\title{
Comparação do Comportamento à Flexão com Restrições à Deflexão de Placas de Compósitos de Matriz Polimérica Epoxídica em Função do Tipo de Fibra de Reforço
}

\author{
Willy A. de Morais, José Roberto M. d'Almeida \\ Departamento de Ciência dos Materiais e Metalurgia, PUC-Rio
}

\begin{abstract}
Resumo: Placas finas de compósitos de matriz polimérica epoxídica reforçada por fibras de vidro, carbono ou aramida, bem como placas de um compósito híbrido vidro-aramida, foram submetidas a ensaios de flexão. As condições de fixação dos corpos de prova foram tais que os ensaios foram realizados com deflexão restrita. Os resultados experimentais para a deflexão máxima dos compósitos puderam ser qualitativamente previstos usando-se o método de balanço de energia de Ritz. Os compósitos reforçados por fibras de carbono apresentaram os maiores valores de energia de iniciação e de força máxima, o que foi atribuído às altas propriedades intrínsecas das fibras. As propriedades específicas do compósito híbrido foram superiores às dos compósitos apenas com fibras de vidro ou fibras aramidas. Este efeito sinergístico indicou um projeto adequado na fabricação deste compósito. O modo de falha macroscópico de todos os compósitos foi semelhante. $\mathrm{Na}$ face frontal, a interação compósito - punção seguiu o comportamento previsto pela Lei de Hertz. Na face posterior, houve preferencialmente delaminação.
\end{abstract}

Palavras-chave: Compósitos de matriz polimérica epoxídica, flexão de placas, matriz de rigidez.

Comparison of the Bending Behavior with Restrained Deflection of Epoxy Matrix Composite Plates as a Function of the Reinforcement Fiber

Abstract: Thin plates of epoxy resin matrix composites reinforced with glass, carbon and aramid fibers, as well as a plate from a hybrid glass-aramid composite, were submitted to flexure tests. The specimens were fixed in order to conduct the tests under restricted conditions of deflection. The experimental results of the maximum deflection agreed qualitatively with the Ritz method of energy balance. Carbon-fiber reinforced composites showed the highest values for the initiation energy and for the maximum force. Both results were attributed to the high intrinsic properties of carbon fibers. The specific properties of the hybrid composite were better than those of the glass or aramid reinforced composites. This synergistic effect is indicative of a properly designed hybrid composite. The macroscopic failure mode of all composites was similar. At the front face the interaction between the composite and the indenter followed the behavior of Hertz Law. At the rear face, delamination occurred preferentially.

Keywords: Epoxy matrix composites, bending of plates, stiffness matrix.

\section{Introdução}

Compósitos de matriz polimérica empregando fibras de vidro, carbono ou aramida como reforço são atualmente usados em inúmeros setores industriais, como por exemplo nas indústrias aeronáuticas, de construção civil e de produtos esportivos ${ }^{[1]}$. Estes materiais aliam a alta resistência destas fibras com a baixa densidade da matriz polimérica. Assim, têm excelentes índices de mérito quando, por exemplo, a massa de uma estrutura ou de um componente é um aspecto relevante do projeto ${ }^{[2,3]}$. Nestas situações, os compósitos apresentam, freqüentemente, um desempenho melhor que os materiais tradicionais de engenharia, tal como as ligas de alumínio. A indústria aeronáutica tem usado amplamente estas características e inúmeras peças e/ou componentes dos modernos aviões de passageiros, bem como de caças supersônicos, são feitas em compósitos de matriz polimérica $^{[1]}$.

Fato comum ao emprego de todos os materiais de engenharia, e dos compósitos, em particular, devido à ortotropia destes materiais, é a necessidade de se caracterizar completamente as suas propriedades fundamentais, tal como o módulo de Young, por exemplo. Entretanto, além das propriedades determinadas em testes padronizados com corpos de prova é, muitas vezes, necessário se testar o componente com suas dimensões reais ou simular em ensaios as condições de contorno reais que o material encontrará em serviço. Por exemplo, restrições à deformação, devido à peça ou componente estar presa a outra parte da estrutura podem prejudicar o desempenho de um material.

Neste trabalho é feita uma análise comparativa do comportamento à flexão de compósitos usados na forma de

Autor para correspondência: José Roberto M. d'Almeida, PUC-Rio, Rua Marques de São Vicente 225, CEP: 22453-900, Rio de Janeiro, RJ. E-mail:dalmeida@dcmm.puc-rio.br 
uma placa fina em posições não-estruturais da fuselagem de aviões, levando em consideração as restrições à deformação impostas pela estrutura. Os aspectos macroscópicos da falha dos compósitos foram determinados e foi feita a correlação entre as propriedades medidas e as características mecânicas das fibras de reforço.

\section{Modelagem do Problema}

O problema a ser analisado pode ser modelado como o de uma placa submetida a flexão e com extremidades fixas, conforme mostrado na Figura 1. Para este caso não existem soluções exatas para resolver as equações constitutivas que governam o problema e soluções aproximadas devem ser empregadas $^{[4]}$. Para placas com ortotropia especial, as soluções usando o método de Ritz de balanço de energia ${ }^{[4]}$ mostram que a máxima deflexão ocorre no centro da placa e é dada pela equação

$$
w_{\text {máx }}=0,00348 \frac{q_{0} a^{4}}{\left[D_{11}+0,6047\left(D_{12}+2 D_{66}\right) R^{2}+D_{22} R^{4}\right]}
$$

onde $q_{0}$ é a carga transversal uniforme aplicada, $a$ é a largura da placa, $R$ é a razão de aspecto da placa (i.e., $a / b)$ e $D_{i j}$ são os termos da matriz de acoplamento flexão-torção. $\mathrm{O}$ momento máximo ocorre na metade de cada aresta e é dado por

$$
M_{\text {máx }}=-0,0513 q_{0} a^{2}
$$

As Equações (1) e (2) mostram que para uma mesma geometria (ou seja, a = constante) e para um mesmo nível de carregamento, o momento atuando sobre a placa será constante independentemente do material [Eq. (2)], mas a deflexão será função das constantes elásticas do compósito ${ }^{[3]}$ pois

$$
D_{i j}=\frac{1}{3} \sum_{k=1}^{n}\left(\bar{Q}_{i j}\right)_{k}\left(z_{k}^{3}-z_{k-1}^{3}\right)
$$

onde $z$ é a coordenada perpendicular ao plano da placa, $k$ é o número de lâminas que formam o laminado e $\bar{Q}_{i j}$ é a matriz de rigidez plana transformada, que é função da matriz de rigidez, $Q_{i j}$, e da orientação, $\theta$, de cada lâmina.

\section{Experimental}

Compósitos reforçados com tecidos de fibras de carbono, vidro ou aramida foram fabricados a partir de lâminas de pré-impregnados (Hexcel Co., Ca.) e foram curados em autoclave, com a utilização de saco de vácuo. Compósitos híbridos vidro-aramida foram fabricados da mesma forma com a configuração $\left[\mathrm{V}, \mathrm{A}_{2}\right]_{\mathrm{s}}$, onde $\mathrm{V}$ e A representam, respectivamente, camadas reforçadas por fibras de vidro e aramida. Resina epoxi foi usada como matriz e o ciclo de cura empregado, recomendado pelo fabricante dos pré-impregnados, é mostrado na Tabela 1 . Na Tabela 2 estão listadas as principais características dos tecidos e das fibras usadas. A Figura 2 mostra uma representação esquemática do sistema experimental usado. Os compósitos aqui analisados foram fabricados com diferentes números de lâminas dependendo do tipo de reforço, de modo a que apresentassem aproximadamente o mesmo nível de resistência ao impacto ${ }^{[5]}$. O número de lâminas empregado foi de 6, 8, 10 e 6 para, respectivamente, os compósitos com fibras de carbono, vidro, aramida e híbrido.

Todos os compósitos fabricados tiveram suas espessuras medidas em vinte diferentes pontos, para se verificar a uniformidade do processo de fabricação e a viabilidade de se usar a espessura média de cada placa para normalizar os valores da carga máxima atingida nos ensaios. As medições foram realizadas com um micrômetro com precisão de $0,001 \mathrm{~mm}$.

A Figura 3 mostra o esquema da montagem utilizada para a realização dos ensaios mecânicos. O conjunto placa e porta-amostras foi posicionado entre os travessões de uma

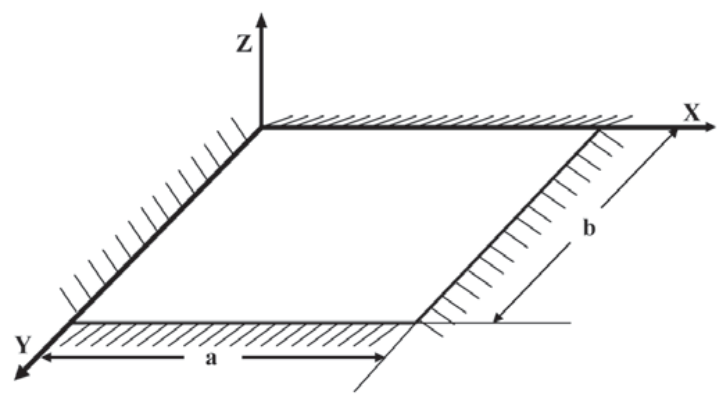

Figura 1. Representação esquemática da placa engastada em suas quatro arestas.

Tabela 1. Ciclo de cura da resina epoxi utilizada como matriz.

\begin{tabular}{cl}
\hline Etapas & \\
\hline $1^{\circ}$ & Aplicação de vácuo (mínimo de $77 \mathrm{kPa}$ ou $0,77 \mathrm{~atm})$ \\
$2^{\circ} \mathrm{o}$ & Aplicação, sob vácuo, de pressão externa de $60 \pm 5 \mathrm{psi}(414 \mathrm{kPa}$ ou $4,1 \mathrm{~atm}) *$ \\
$3^{\circ}$ & Aquecimento até $177 \pm 5^{\circ} \mathrm{C}$, sob taxa de aquecimento de 0,5 a $2,7{ }^{\circ} \mathrm{C} / \mathrm{min}$ \\
$4^{\circ}$ & Manutenção de $60 \pm 5 \mathrm{psi}(414 \mathrm{kPa} \text { ou } 4,1 \mathrm{~atm})^{*}$ e $177 \pm 5^{\circ} \mathrm{C}$ por 120 a $135 \mathrm{~min}$ \\
$5^{\circ}$ & Resfriamento para temperatura inferior a $65^{\circ} \mathrm{C}$, sob pressão, a uma taxa de $2,7^{\circ} \mathrm{C} /$ min no máximo \\
\hline
\end{tabular}

* Para o compósito de fibra de carbono a pressão é de $100 \pm 5$ psi (689 kPa ou 6,8atm). 
Tabela 2. Características dos tecidos e propriedades das fibras de reforço.

\begin{tabular}{lccc}
\hline & Carbono & Vidro & Aramida \\
\hline Trançado & Eight hardness & Eight hardness & Crowfoot \\
Peso do tecido, $\mathrm{g} / \mathrm{m}^{2}$ & $370 \pm 20$ & $303 \pm 10$ & $170 \pm 10$ \\
Número de filamentos por $\mathrm{cm}^{2}$ & $9,5 \times 9,5$ & $22,4 \times 21,2$ & $7 \times 7$ \\
Módulo de Young, GPa * & 231 & 70 & 131 \\
Tensão de ruptura, $\mathrm{MPa} *$ & 3654 & 1750 & 3792 \\
\hline
\end{tabular}

*Valores das fibras.

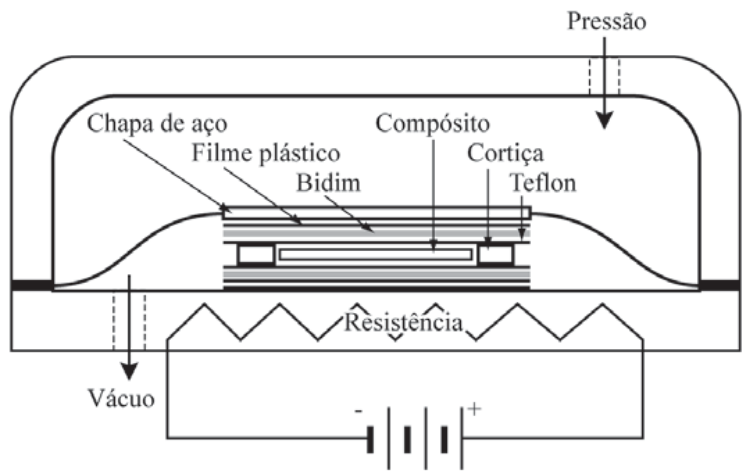

Os pré-impregnados são envolvidos por dois tecidos de materiais antiaderente (Teflon $®)$, dois tecidos de material absorvente (Bidim $\left.{ }^{\circledR}\right)$, dois filmes plásticos e duas chapas de aço. As laterais são protegidas por cortiça.

Figura 2. Configuração do empilhamento das lâminas dentro da autoclave.

máquina universal de ensaios Instron, com célula de carga com $10 \mathrm{kN}$ de capacidade, de forma que a base do porta amostras permaneceu rigidamente apoiada sobre a mesa da máquina de testes. A Figura 4 mostra em detalhe o porta amostras usado. Este dispositivo foi preparado de modo a garantir que as bordas da placa em teste não escapem do sistema de fixação durante a aplicação da carga, conforme descrito na literatura ${ }^{[6]}$. O punção usado tinha ponta esférica, com diâmetro de $1 / 2$ polegada, sendo o carregamento realizado no centro geométrico de cada placa. Os ensaios foram realizados a uma taxa de carregamento de $2 \mathrm{~mm} / \mathrm{min}$. Os parâmetros obtidos do ensaio foram:

$\mathbf{F}_{\mathrm{D}}$ : nível de força na qual o compósito começa a apresentar dano macroscópico, que é perceptível por uma queda no gráfico de força $v s$. deslocamento.

$\mathbf{F}_{\text {máx: }}$ : força máxima que o compósito suporta durante o ensaio.

$\Delta \mathbf{L}_{\mathbf{D}}$ : nível de deslocamento do travessão da máquina de ensaio no qual ocorre o início da geração dos danos macroscópicos no compósito.

$\Delta \mathbf{L}_{\text {Fmáx }}$ : nível de deslocamento do travessão da máquina no qual é atingida a força máxima.

$\Delta \mathbf{L}_{\text {frat }}$ : deslocamento total do travessão da máquina ao término do ensaio com a ruptura da amostra testada.

$\mathbf{E}_{\mathrm{T}}$ : energia total consumida, calculada como sendo a área sob as curvas de força $v s$. deslocamento.

$\mathbf{E}_{\mathbf{I}}$ : energia de iniciação, que é o valor de energia até o início da geração de danos macroscópicos.

$\mathbf{E}_{\mathbf{p}}$ : energia de propagação, valor de energia associado à propagação dos danos até a ruptura final da amostra $\left(\mathrm{E}_{\mathrm{P}}=\mathrm{E}_{\mathrm{T}}-\mathrm{E}_{\mathrm{I}}\right)$.

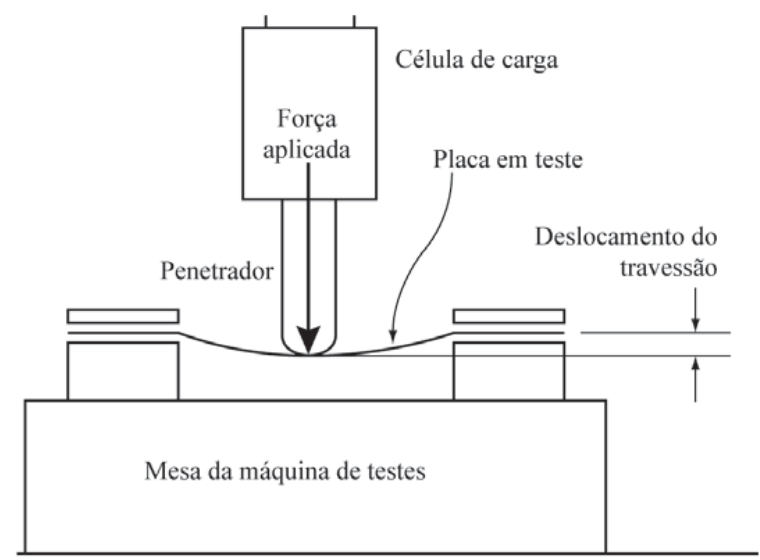

Figura 3. Esquema da montagem utilizada para os ensaios.

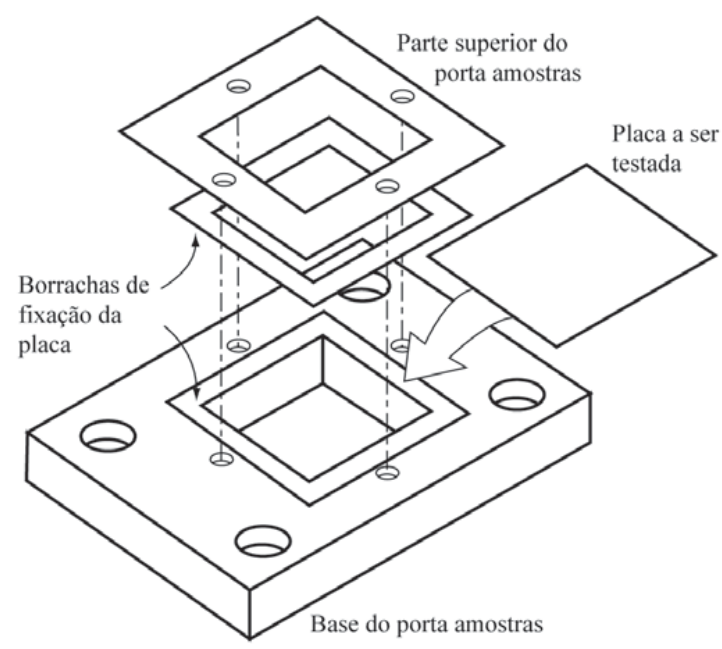

Figura 4. Dispositivo para fixação dos corpos de prova.

A microestrutura dos compósitos foi analisada por microscopia óptica para verificar a distribuição das fibras, presença de áreas ricas em resina, bolhas e trincas. A partir das imagens coletadas foi realizada, usando-se análise digital de imagens, a determinação da fração volumétrica de fibras e de vazios, bem como se determinou o diâmetro médio das fibras. O procedimento usado para estas determinações quantitativas está descrito em outro trabalho ${ }^{[7]}$.

\section{Resultados e Discussão}

Os valores médios e os respectivos desvios padrão relativos à variação da espessura das placas fabricadas são mos- 
trados na Tabela 3. Um gráfico representativo da variação das medidas em função do comprimento das placas para os laminados com fibras aramidas é mostrado na Figura 5. Resultados semelhantes foram obtidos para as demais placas reforçadas com as diversas fibras. Pode-se observar que não houve variação significativa dos valores obtidos para todos os sistemas, indicando placas com espessura uniforme. Desta forma o valor médio mostrado na Tabela 3 pode ser usado como referência da espessura de cada laminado.

A análise digital de imagens mostrou que a fração volumétrica de fibras, $V_{F}$, e de vazios, $V_{v}$, variou muito pouco entre os compósitos. A fração de fibras está na faixa $0,55<V_{F}<0,65$ e a de vazios $V_{v}<0,02$. O valor para os vazios é particularmente interessante pois atende as especificações de materiais aeronáuticos ${ }^{[8]}$. O valor do diâmetro médio medido para as fibras de carbono, aramida e

Tabela 3. Espessura média dos laminados testados.

\begin{tabular}{lcc}
\hline Compósito & Número de camadas & Espessura $(\mathbf{m m})$ \\
\hline Carbono & 6 & $2,29 \pm 0,002$ \\
Vidro & 8 & $1,92 \pm 0,03$ \\
Aramida & 10 & $2,24 \pm 0,06$ \\
Híbrido & 6 & $1,34 \pm 0,09$ \\
\hline
\end{tabular}

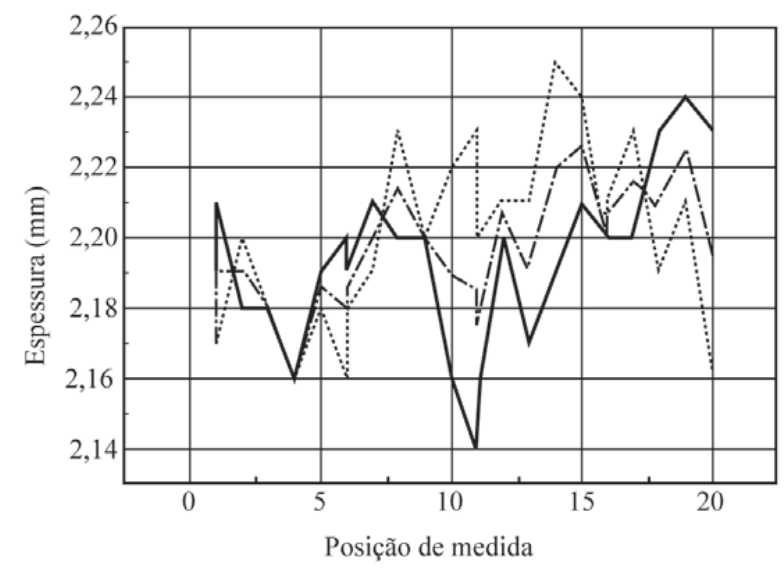

Figura 5. Variação da espessura das placas ao longo de seu comprimento. São mostrados os traços das curvas obtidas para 3 placas distintas. vidro foi de 12, 24 e $8 \mu \mathrm{m}$, respectivamente.

A Figura 6a mostra uma microestrutura típica observada, onde se deve destacar a baixa fração de vazios e a presença de camadas ricas em resina entre as camadas de fibras. Estes aspectos microestruturais foram comuns a todos os compósitos. Na Figura $6 \mathrm{~b}$ é apresentada com maior detalhe a região dentro de uma mecha de fibras.

Os resultados obtidos nos ensaios mecânicos são mostrados nas Tabelas 4 e 5. Na Tabela 4 é apresentada também a força específica calculada dividindo-se $F_{D}$ e $F_{\text {máx }}$ pela espessura média de cada placa. Na Tabela 5 estão calculadas as energias específicas. Na Figura 7 são apresentadas as curvas carga $v s$. deslocamento características para cada compósito. Pode-se observar que o compósito com fibras de carbono apresentou o melhor desempenho tanto em relação aos valores absolutos de força, $\mathrm{F}_{\mathrm{D}}$ e $\mathrm{F}_{\text {máx }}$, quanto em relação aos valores relativos de força por unidade de espessura. Este compósito apresentou também os maiores valores absolutos e específicos de energia total, $\mathrm{E}_{\mathrm{T}}$, e de energia de iniciação, $\mathrm{E}_{\mathrm{I}}$. $\mathrm{O}$ valor de $\mathrm{E}_{\mathrm{I}}$ será considerado aqui como sendo representativo da resiliência dos compósitos, pois representa a parcela de energia de deformação absorvida na região onde, ao menos macroscopicamente, não houve qualquer geração ou propagação de defeitos.

Todos estes resultados podem ser atribuídos diretamente às propriedades intrínsecas das fibras de carbono (maior módulo de elasticidade e tensão de ruptura elevada, Tabela 2), o que, por outro lado, contribui para as menores deflexões obtidas para os compósitos de fibra de carbono conforme previsto pela Equação (1). O traço da curva carga $v s$. deslocamento deste compósito mostra um comportamento característico de materiais de alta resistência, apresentando uma queda brusca após o ponto de carga máxima, Figura 7a. Entretanto, devido ao entrecruzamento das fibras no tecido, a fratura não é frágil ${ }^{[9]}$ e não ocorre subitamente após o ponto de carga máxima, como observado para compósitos unidirecionais onde a energia é praticamente toda gasta no carregamento elástico do compósito ${ }^{[10]}$. De fato, como apresentado na Tabela 5, uma parcela substancial de energia foi gasta na propagação antes da falha final do compósito de fibra de carbono.
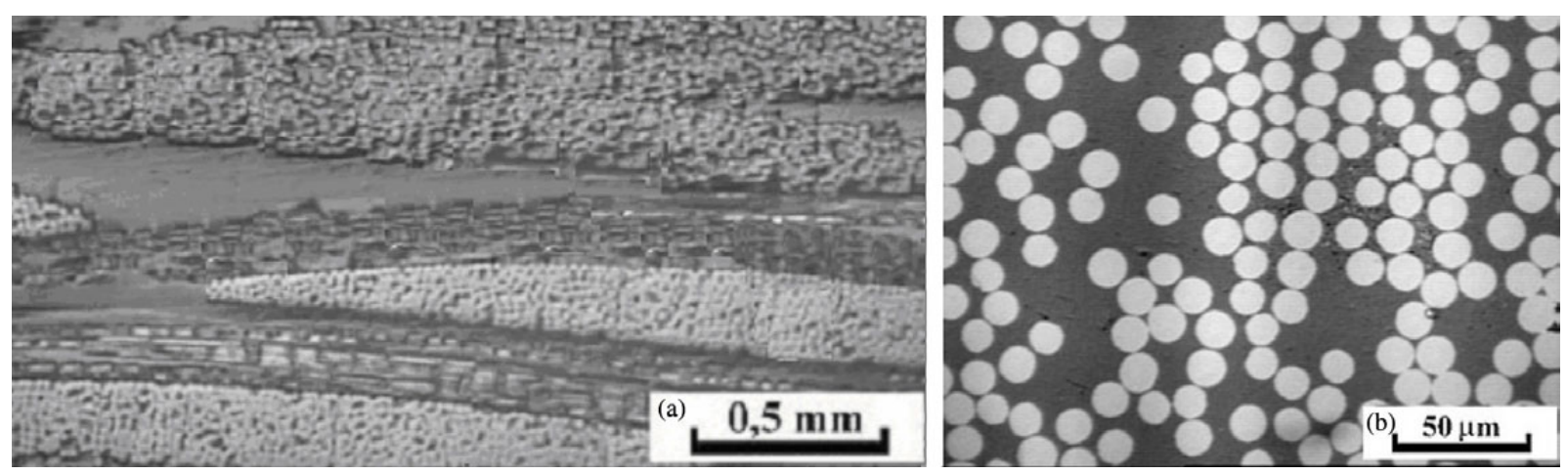

Figura 6. Aspectos micrográficos comuns aos compósitos. a) Baixa fração de vazios. Compósito híbrido, b) Fibras relativamente bem distribuídas na matriz. Compósito de carbono. 
Tabela 4. Resultados experimentais dos ensaios mecânicos.

\begin{tabular}{lccccccc}
\hline Compósito & \multicolumn{2}{c}{ Força (kN) } & \multicolumn{2}{c}{ Força Específica (N/m) } & \multicolumn{3}{c}{ Deslocamento (mm) } \\
& $\mathbf{F}_{\mathbf{D}}$ & $\mathbf{F}_{\text {máx }}$ & $\mathbf{F}_{\mathbf{D}}$ & $\mathbf{F}_{\text {máx }}$ & $\mathbf{\Delta L}_{\mathbf{D}}$ & $\mathbf{\Delta L}_{\mathbf{F m a ́ x}}$ & $\mathbf{\Delta L}_{\text {frat }}$ \\
\hline Carbono & 2,63 & 3,11 & 1,15 & 1,36 & 5,5 & 6,0 & 11,2 \\
Aramida & 1,96 & 2,33 & 0,86 & 1,02 & 6,0 & 6,9 & 12,5 \\
Vidro & 1,44 & 1,63 & 0,75 & 0,85 & 6,4 & 7,7 & 13,0 \\
Híbrido & 1,22 & 1,37 & 0,91 & 1,02 & 6,7 & 7,6 & 12,1 \\
\hline
\end{tabular}

Tabela 5.Valores experimentais das energias total, de iniciação e propagação de defeitos. Valores absolutos (J). Valores específicos (kJ/m).

\begin{tabular}{lcccccc}
\hline Compósito & \multicolumn{2}{c}{ Energia Total } & \multicolumn{2}{c}{ Energia de Iniciação } & \multicolumn{2}{c}{ Energia de Propagação } \\
& Absoluta & Específica & Absoluta & Específica & Absoluta & Específica \\
\hline Carbono & 20,32 & 8,87 & 7,23 & 3,16 & 10,99 & 4,80 \\
Aramida & 19,23 & 8,58 & 5,88 & 2,63 & 10,75 & 4,80 \\
Vidro & 11,36 & 5,92 & 4,61 & 2,40 & 6,75 & 3,52 \\
Híbrido & 8,99 & 6,71 & 4,09 & 3,05 & 4,90 & 3,66 \\
\hline
\end{tabular}

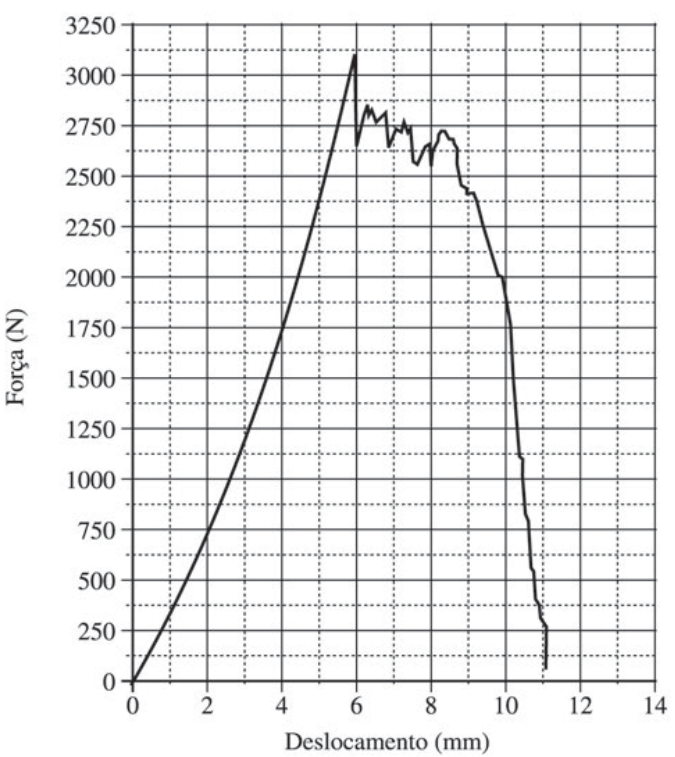

(a)

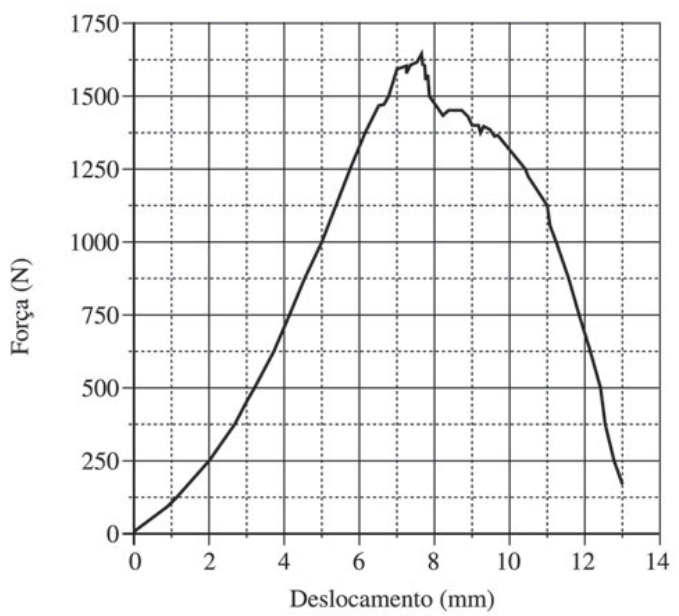

(c)

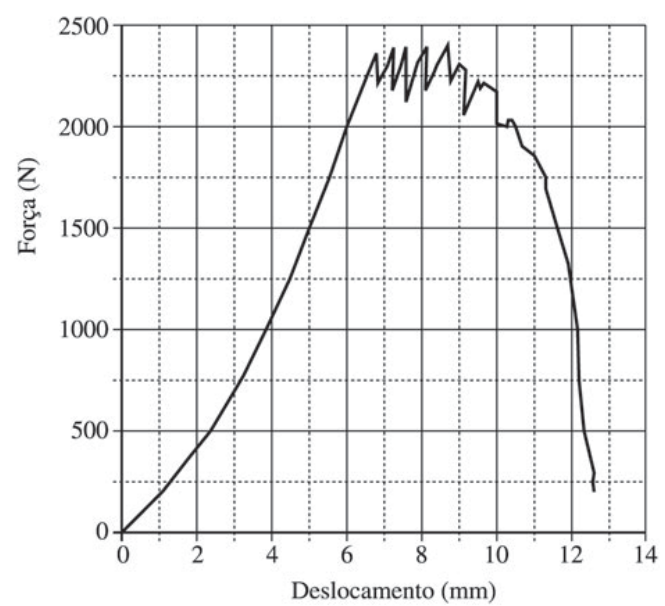

(b)

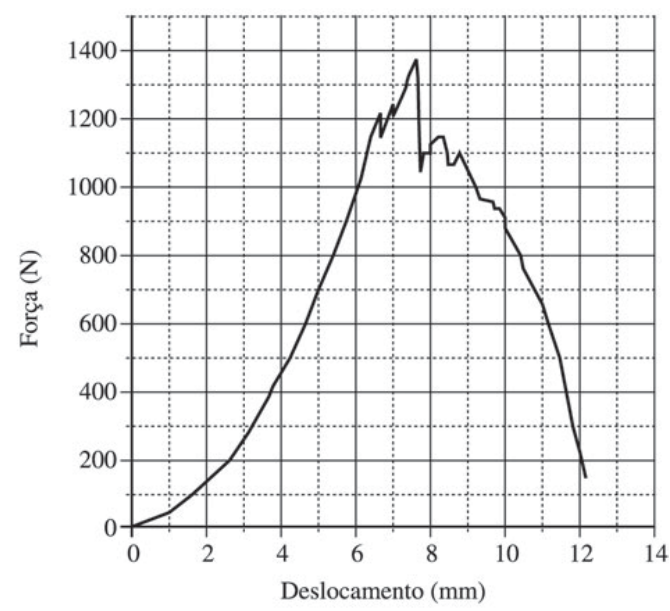

(d)

Figura 7. Traço representativo das curvas carga vs. deslocamento dos compósitos. a) Fibra de carbono, $b$ ) Fibra aramida, $c$ ) Fibra de vidro, $d$ ) Híbrido. 

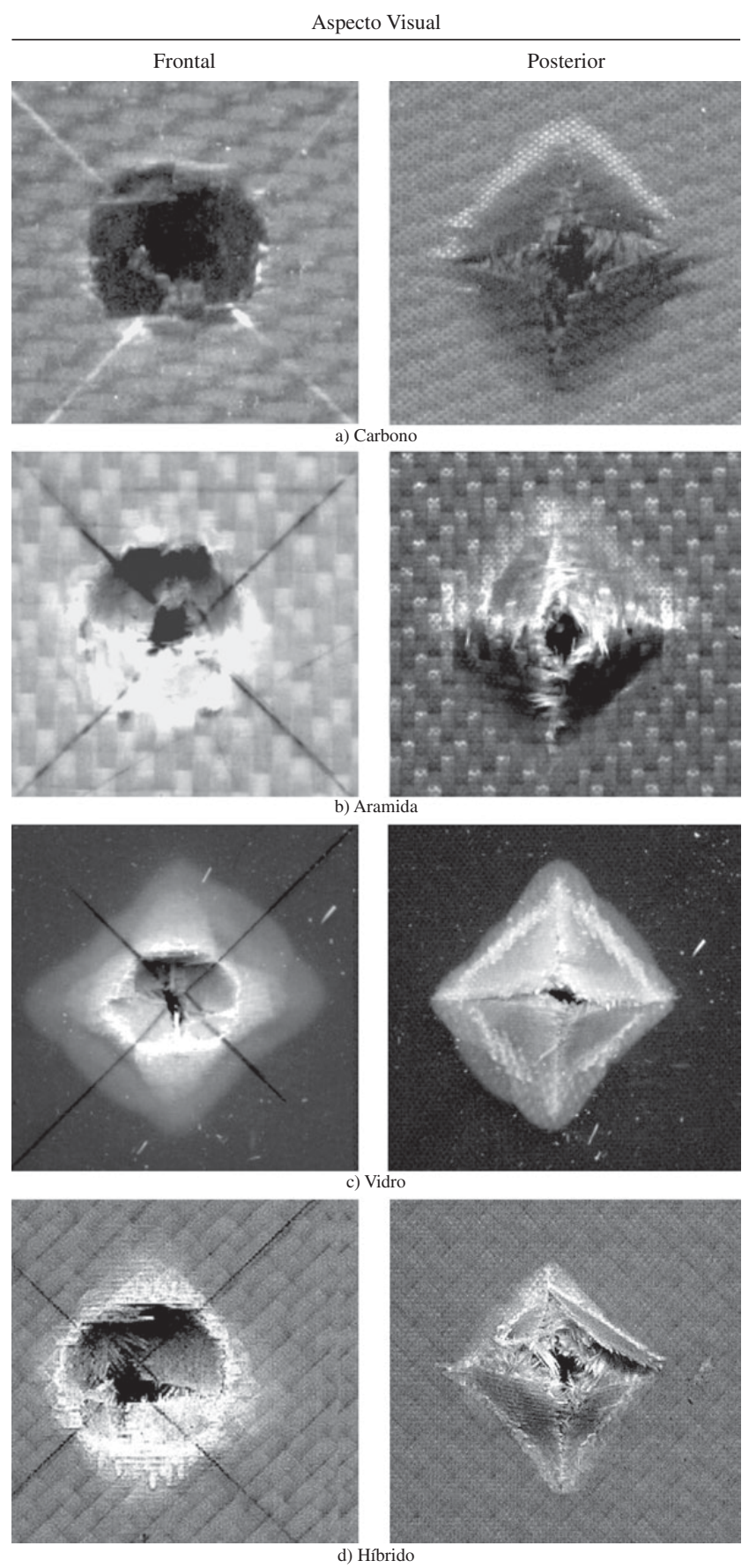

Figura 8. Aspecto macroscópico da fratura dos compósitos.

Na Figura 8a é mostrada a fratura macroscópica observada para o compósito com fibra de carbono. Na face frontal, a indentação circular observada reflete o trincamento da matriz e o subsequente rompimento das interfaces e fibras ${ }^{[1]]}$. Neste tipo de falha a interação entre o material e o punção segue o comportamento previsto pela Lei de Hertz ${ }^{[1,12]}$, e indica que está ocorrendo uma deformação homogênea no material ${ }^{[9]}$. Na face posterior, a fratura macroscópica tem uma forma de losango cujos eixos coincidem com a direção das fibras. Este comportamento é indicativo de delaminação e trincamento das interfaces fibra-matriz nas faces sob maior tensão trativa ${ }^{[11]}$.

Conforme mostrado na Figura 8, todos os compósitos testados mostraram o mesmo tipo de falha macroscópica. Desta forma, os resultados dos ensaios mecânicos podem ser diretamente comparados entre si pois os eventos de falha foram semelhantes, tanto no tipo quanto em sua seqüência.

O comportamento do compósito de aramida é caracterizado por uma parcela de energia de propagação bem maior do que a de energia de iniciação (Tabela 5). Esta característica dos compósitos reforçados por aramidas é devida, em parte, aos diversos mecanismos absorvedores de energia proporcionados pelas fibras em si, a saber ${ }^{[13]}$ : formação de bandas de deformação nas próprias fibras (kink bands) e descascamento superficial das fibras (peeling). Esta última característica em particular é responsável por delaminação, o que contribui para aumentar a parcela de energia de propagação destes compósitos ${ }^{[10]}$. Este aspecto está retratado pela seqüência de serrilhas com carga máxima praticamente constante na curva carga $v s$. deslocamento deste compósito, Figura $7 b$. Conforme previsto pela Equação (1), a deflexão deste compósito foi maior do que a do compósito reforçado por fibra de carbono.

A Figura $7 \mathrm{c}$ apresenta a curva força $v s$. deslocamento para o compósito com fibra de vidro. O comportamento mecânico obtido foi bem distinto daquele verificado para as placas reforçadas com fibras de carbono e aramida. O processo de ruptura do compósito reforçado por fibras de vidro é caracterizado por uma pequena perda de resistência no ponto de carga máxima, seguida por uma redução gradual do nível de carregamento suportado até a ruptura final. A parcela de propagação deste compósito ainda foi superior à de iniciação, porém seus valores são os menores entre os materiais analisados. Da mesma forma, este compósito apresentou a menor força máxima, porém mostrou uma maior deflexão até o início da geração de danos, $\Delta \mathrm{L}_{\mathrm{D}}$, e até a sua ruptura final, $\Delta \mathrm{L}_{\text {frat }}$ em relação aos compósitos de carbono e aramida (Tabela 4). Este comportamento está de acordo com o predito pelo modelo descrito pela Equação (1), tendo em vista as menores constantes elásticas das fibras de vidro, e consequentemente de seu compósito, em relação às fibras de carbono e aramidas. Deve-se lembrar que a fração volumétrica de todos os compósitos aqui analisados foi mantida constante, dentro do erro experimental.

O compósito híbrido apresentou o comportamento mais frágil dentre os compósitos analisados. A curva carga $v s$. deslocamento deste compósito apresentou uma queda brusca da carga após o ponto de carga máxima, conforme mostrado na Figura 7d. De fato, para este compósito a parcela de energia de propagação é praticamente igual à parcela de energia de iniciação (cerca de 5\% maior) (Tabela 5). Além disto, a energia de iniciação específica deste compósito foi bastante elevada, sendo inferior apenas ao valor da energia do compósito com fibras de carbono. A análise de todos os resultados obtidos para este compósito indicam, entretanto, que há uma forte sinergia entre as fibras usadas. Pode-se 
observar nas Tabelas 4 e 5 que, notadamente na região elástica, os valores das propriedades específicas do compósito híbrido foram superiores aos valores dos compósitos individuais com fibras de vidro e com fibras aramidas. Este efeito está bem reportado na literatura ${ }^{[14]}$ e é indicativo da boa arquitetura de um compósito híbrido ${ }^{[15]}$. De fato, devido à diferença no módulo elástico entre a fibra de vidro e a aramida, as fibras de vidro podem se defletir mais e, assim, transferir para si parte da carga suportada pelas aramidas após estas se romperem. Esta transferência de carga retarda a falha dos compósitos híbridos ${ }^{[15]}$.

\section{Conclusões}

Conforme bem estabelecido na literatura, o desempenho mecânico dos compósitos avaliados variou em função do tipo de fibra empregado como reforço. Sob as condições de carregamento e fixação dos corpos de prova empregadas neste trabalho, o compósito reforçado por fibras de carbono apresentou o melhor desempenho em termos de energia e de força máxima. Entretanto, este compósito apresentou os menores valores de deflexão. Todo este comportamento foi atribuído às propriedades intrínsecas das fibras de carbono. Qualitativamente, a deflexão máxima apresentada pelos compósitos pode ser prevista usando-se o modelo de balanço de energia de Ritz.

O compósito híbrido vidro-aramida apresentou propriedades superiores aos compósitos reforçados apenas com fibras de vidro ou aramida. As propriedades específicas deste compósito, principalmente em termos da energia de iniciação $(\approx$ resiliência) tornam-no atrativo como um substituto mais barato que o compósito de fibras de carbono.

O comportamento do compósito de aramida foi caracterizado por uma parcela de energia de propagação superior à parcela de energia de iniciação. Este comportamento foi explicado em termos dos diversos mecanismos absorvedores de energia proporcionados pelas fibras aramidas.

A falha macroscópica de todos os compósitos foi semelhante, sendo descrita pela Lei de Hertz na superfície frontal, onde o punção entra em contato com as placas. A falha na face posterior foi dominada por delaminações que foram atribuídas à presença de tensões trativas.

\section{Agradecimentos}

Os autores agradecem o CNPq pelo apoio financeiro e ao CTA-AMR pelo auxílio na fabricação dos compósitos.

\section{Referências Bibliográficas}

1. Gibson, R.F. Principles of Composite Materials Mechanics, McGraw-Hill, New York, 1994.

2. Ashby, M.F. Criteria for selecting the components of composites, Acta Metall. Mater., 41, p. 1313-1335, 1993.

3. Ferrante, M. Seleção de Materiais, Editora UFSCar, São Carlos, 1996.

4. Whitney, J.M. Structural Analysis of Laminated Anisotropic Plates, Technomic Pu. Co., Lancaster, PA, 1987.

5. Morais, W.A. Estudo e caracterização da resistência a impactos de baixas energias de materiais compósitos, Tese de Mestrado, Departamento de Ciência dos Materiais e Metalurgia, PUC-Rio, Rio de Janeiro (1999).

6. Wardle, M.W.; Tokarsky, E.W. Drop weight impact testing of laminates reinforced with kevlar aramid fibers, Eglass, and graphite, Composites Technol. Rev., 5, p. 410, 1983.

7. Paciornik, S.; Martinho, F.M.; Mauricio, M.H.P.; d'Almeida, J.R.M. Analysis of the mechanical behavior and characterization of pultruded glass fiberresin matrix composites, Comp. Sci. \& Technol., 63, p. 295-304, 2003.

8. Purslow, D. On the optical assessment of the void content in composite materials, Composites, 15, p. 207-210, 1984.

9. Park, R.; Jang, J. Effect of laminate geometry on impact performance of aramid fiber/polyethylene fiber hybrid composites, J. Appl. Polym. Sci., 75, p. 952959, 2000.

10. Naglis, M.M.M.; d'Almeida, J.R.M. Avaliação dos Modos de Falha sob Impacto de Compósitos de Matriz Polimérica Reforçados por Fibras, Polímeros: Ciência e Tecnologia, 8, p. 54-60, 1998.

11. Hou, J.P.; Petrinic, N.; Ruiz, C.; Hallett, S.R. Prediction of impact damage in composite plates, Comp. Sci. \& Technol., 60, p. 273-281, 2000.

12. Goldsmith, W. Impact: the theory and physical behaviour of colliding solids, Edward Arnold Publishers Ed. Ltd., London, 1960.

13. de Sousa M.V.; Monteiro, S.N.; d'Almeida, J.R.M. Fractographic Analysis of Hybrid Glass FabricAramid Fabric Epoxy Matrix Composites. Part II: Aramid-Fabric Topographic Features. Proceedings of the XVIII Congress of the Brazilian Society for Microscopy and Microanalysis, Águas de Lindóia, 2831 de Outubro, Acta Microscopica Supplement A, p. 125-126, 2001.

14. Summerscales, J.; Short, D. Carbon fibre and glass fibre hybrid reinforced plastics, Composites, 9, p. 157-166, 1978.

15. Horton, R.E. Damage Tolerance of Composites, in Engineered Materials Handbook - Vol. 1, Composites, Reinhart TJ, ed., ASM International, Materials Park, OH, p. 259-267, 1987.

Recebido: $22 / 01 / 2003$

Aprovado: 22/04/2003 